\title{
Research on the Application of Cloud Desktop Technology in Laboratory in Colleges and Universities
}

\author{
Jinjiang $\mathrm{Gu}$ \\ Jiangsu Institute of Commerce, Jiangsu, Nanjing, 211168, China
}

Keywords: Cloud desktop technology, University, Computer, Laboratory

\begin{abstract}
With the continuous development of computer technology, colleges and universities invest a lot of money to update the computer almost every year, but the effectiveness of the use of computer is still low, even it is difficult to meet the actual needs of teaching. In order to improve the teaching effect of computer laboratory in colleges and universities, it is urgent to introduce cloud desktop as a new technology appears in recent years. On the basis of defining related concept of cloud desktop technology, this paper analyzed the current situation of computer laboratory in colleges and universities, and discussed the method and effect of cloud desktop technology used in college laboratory.
\end{abstract}

\section{Introduction}

Colleges and universities pay much attention to the construction of computer laboratories and continue increasing investment. Good management of lab equipment is crucial to improve the usage rate of laboratory in the condition of no increase in the amount of laboratory staff. Cloud desktop technology was born to put forward a new solution to the information construction in the world. The use of cloud desktop technology can easily solve the previous problems appeared in the process of computer laboratory operation such as repetitive input, difficult equipment maintenance, unreasonable resource allocation and so on. How to reasonably use the cloud desktop technology to create the modern computer laboratory is one of the most important topics in the field of computer teaching in colleges and universities.

\section{Definition of related concepts of cloud desktop technology}

Cloud technology, also known as cloud computing, mainly refers to a new technology developed by traditional computing technology such as distributed computing, grid computing and parallel computing and modern network technology. Cloud technology takes the network as the main carrier, forming a extremely powerful computing system, relying on the modern business pattern such as Iaas, PaaS and SaaS, and distributing so powerful computing capacity to every user terminal through the effective combination of various types of low cost entities. The core thought of cloud computing is to continuously improve the ability of cloud processing, and then the burden of the user terminal processing can be reduced, and the user terminal can become an input and output device, and as a carrier to share the ability of cloud computing.

As a new type of large scale computing, cloud computing fully takes data as the core, is a data intensive computing mode. It has its own unique technology in various fields, such as, data storage, management, programming and virtualization technology, etc., and cloud desktop technology is just an important part of virtualization technology. The technology uses lean clients to replace the traditional personal computers, and powerful server cluster to be applied. After using the virtualization technology, all kinds of applications will be changed as centralized services, in order to fully realize the great innovation in the form of the deployment, we should deliver on time.

\section{Analysis on the current status of computer lab in colleges and universities}

a. Arrangement and update of computer equipments are time and labor consuming. In order to guarantee the college students'computer needs from different major in teaching, training, 
experimental assessment and other aspects to be fully met, computer lab managers need to put a number of operating systems and all kinds of software layout and installation in every computer lab, and often have to spend a lot of time and energy. At the same time, lab managers also need to spend a lot of energy to implement computer lab maintenance work, the main content covers the update, upgrade, patch of software, etc..

b. Computer lab is lack of complete unified hardware facilities. Because the equipment of computer lab upgrading year by year, causing all kinds of different configurations of computers concentrated in same laboratory. It is not easy to well manage and maintain all the computers of the lab, the traditional network scheme is often difficult to work.

c. The problem of authorization restrictions of software terminals is existence universally. Professional software often required to bind and activate a variety of terminals, register and activation and some other operations must be done in every computer in the process of installation of the official version of software, which is very complicated, and the cost of software is very high. There are legal and security risks in using cracked version of software.

d. The network platform is lack of adequate risk defense capabilities. Because of the network attacks, a few learners download or access to the unsafe site, which easily causes the problems of central network, and even affects the daily teaching and management.

e. Paying attention to the management of students' daily behavior of computer operating in teaching. If the teachers in the process of experimental teaching can not monitor the behavior of computer operating of the students real-timely, they can only know the learning situation through the students' final grades, so it is difficult to guarantee the effectiveness of experimental teaching. At the same time, the lab managers are not able to have a comprehensive and in-depth understanding of the students' specific behavior of computer operating. It will be very difficult to locate the person in charge once the computer room has an accident.

f. The distribution of machine-position of the teachers is extremely decentralized. Because the machine-position of computer is often not concentrated, scattered in all of the rooms, so the workload is greatly enhanced caused by the update and maintenance of the teacher machine, lab managers have to update one by one, the working intensity is quite large.

\section{The method of cloud desktop technology applied to the laboratory in colleges and universities.}

a. Computer software can be installed at any time, and the update of the patch will not lead to the nterruption, so it will not affect normal teaching. After determination of the computer laboratory teaching environment, if you want to update later, you do not need to wait for no class to install software, you can install, uninstall software and change configuration and some other operations at any time, even some operations like maintenance and update of the cloud desktop that is still in use, the teachers' normal teaching activities will not be affected, and as long as the newly installed software exits from the current cloud desktop, then you can use it immediately when it enters again.

b. Reasonable management and more flexible allocation must be done to the authorization of legitimate software, so the cost of software purchasing will be reduced. In terms of the cost of software procurement in colleges and universities, allocation of some of the professional quality of simulation software and design software, etc., should be based on the specific requirements of the teaching. Because the cost of the legitimate software is very high, as well as the cost of subsequent maintenance, after hardware virtualization technology is applied to cloud desktop technology, it will solve the problem of the license needed to activate every computer in the process of installation, only an activition on the server-side, mass use can be done on the client-side.

c. Multiple batches of software can be installed at one time. Computer lab administrators can be connected with the cloud desktop in cloud servers, then it can be the corresponding software on cloud desk. It has no difference with the process installing new software for the machine, and multiple batches of computers installed with new software in computer labs can be immediately put into use, which belongs to using after installing, no need to download the image and carry out network cloning. 
At the same time, teachers' computer can be installed naturally which distributed in different floors and classrooms.

d. Different versions of software of the computer can be installed and run at the same time, so the teachers' habits can be met. Since teachers and courses are different, the demand of software version may be different. In the past, this situation noly could be accommodationist, which wuold affect the teaching effects, but when the cloud desktop technology is applied, you can install software on a variety of different types of cloud desktop, and then the problem will be solved truly.

Strictly controling and managing learners' behavior, so that we can mannage the specific operation of them more strictly. Teachers will forbid the students to access computer by CD-ROM and U disk after doing necessary settings. You can effectively control the online behavior of the learners while setting up the white list of the process and network access, once the software they use has no relationship with teaching, will they be disabled by administrators. Owing to the above control functions, learners are lack of the opportunity to chat and play games, they have to learn the contents the teachers teach in the classroom seriously. The teachers can also carry out more relaxed and harmonious teaching, and can ensure the safety of computer lab network as well as prevent the virus spread everywhere.

e. Administration of remote computer is more flexible and convenient. Adminitrators of computer labs can do remote operation to computers within the scope of the management. Specifically, adminitrators can use a wake-up function the remote network have to start the computers in the lab in a remote operation before maintaining all kinds of data or teaching; they can turn off the computers by remote functions after class. After class of one class, the computer system needs to be switched, restarting the execution link of computers by remote operation, so that different systems can be switched in time. The time of the computer system can be synchronized through remote operation as some computers restore factory time because of the power down of computer motherboard or artificially modified,etc.. In the condition of no need to copy data, a few computer IP address can be amended through remote operation.

f. The function of computer operating inquiry and monitoring can ensure the effectiveness of teaching. The system can automatically record the computer operating of the learners, for example, the time of access to the site and the application of various procedures, etc., and the computer name v time and operating show in the computer system management-side. Administrators and teachers of computer labs belonging to the management-side can fully monitor the operation of the learners, and effectively master the complete process of the experiment. At the same time, the computer records of the learners will be kept in the database log mode. Based on account rights, the administrators and teaching staff of computer lab can be queried. Once the computer lab has an accident, after the students have access to a bad site, the administrator can query the computer log file, and then quickly locate, and find the responsible person. Using the cloud desktop technology to manage the computer lab can be a good way to improve the maintenance of computer room. The single computer running speed must not lower than the traditional form of management, and can better integrate various types of computers to be unified management. To improve the defensive capabilities of the local system, ignoring the hardware and system environment configuration of the client-side, making different cloud desktop files for various types of software, so it can be a good solution to the previous question to install again and again.

\section{The effect of cloud desktop technology applied to the lab in colleges and universities.}

a. The efficiency of equipment utilization in university computer lab has been improved. Cloud desktop technology can fully support implementing the unified management of heterogeneous computers, so in this way all kinds of different batches of computers can be centralized used, to achieve the maximum use of the old computer. I think that the use of the current server can absolutely set the cloud desktop server, the maximum potential of the current server can exerted. Cloud desktop technology uses virtualization solutions, the requirements for the network bandwidth are quite low, 
can keep the switches and other facilities of computer lab. It does not need to upgrade the corresponding network, so as to greatly enhance the using effect of network equipment.

b. The efficiency of management is improved effectively. Teachers' computer can be dispersed by physical method, you can manage them in a unified and centralized way and mainten all kinds of updated software. Once you have a problem, you can update it on the administration. Then unified distribution should be done, all terminal computers can use updated software and patches. As a result, the software can be installed at any time without affecting the teaching. The effect of teaching can be improved by fast customization of the targeted teaching environment and the settlement of the conflict and compatibility of software.

c. Make sure that the experimental teaching is not affected. If the network is abnormal or the server failure, the cloud desktop can continue working and will not disconnect, and even can switch to the computer for offline operation, in order to In order to guarantee the process of teaching has the continuity, so as to affect the process of teaching because of the failure of the equipment and network.

d. Have a higher data and network security. As all the software is retained in the server, the state can be automatically restored after the cloud desktop closes, so the security and stability can be made sure, will not be destroyed. The security problem of the local computer system can be well solved by the active defense and automatic recovery technology.

e. Be able to reduce the scale of investment effectively. With cloud desktop technology, you can directly use the current server, so you do not need to invest money in purchasing new servers or transforming the existing network environment, and then you can save the corresponding cost.

\section{Conclusion}

In general, cloud desktop technology is a new computer resource utilization model. Compared to the traditional model, it is not only simple and flexible but also more economical and practical. The learners can use the computer network resources as freely as the daily use of water and electricity, really realize to use according to the requirements and to pay according to the real. Laboratories in colleges and universities also need to use this model, so that the cloud desktop technology can be better applied to the university laboratory, and thus promoting the university computer teaching and research to exert more important value.

\section{Acknowledgments}

This paper is the key project of Jiangsu Institute of Commerce, Issue name: Research on cloud desktop technology based on VM container, project number: JSJM014.

\section{References}

[1] Yanhua Li. Overview of research status of cloud computing technology. Computer knowledge and technology. 2009(22)

[2] Yuanshun Dai. Brief description of cloud computing technology. Information communication technology, 2010(2).

[3] Jingjing Cheng. Research and design of cloud desktop technology scheme based on virtualization. Communication technology of Guangdong, 2011(6)

[4] Ning Han. Application of cloud desktop technology in the construction and teaching of college information. Education of modern enterprise, 2012(5)

[5] Fang Wang. Analysis and research of cloud computing. Information technology, 2013(6)

[6] Miaoli Wu. Construction of laboratory resource sharing platform based on cloud desktop Technology. Laboratory research and exploration, 2014(12) 